\title{
Automatic Stave Discovery for Musical Facsimiles
}

\author{
Radu Timofte ${ }^{1}$ and Luc Van Gool ${ }^{1,2}$ \\ ${ }^{1}$ ESAT-VISICS /IBBT, Catholic University of Leuven, Belgium \\ ${ }^{2}$ D-ITET, ETH Zurich, Switzerland
}

\begin{abstract}
Lately, there is an increased interest in the analysis of music score facsimiles, aiming at automatic digitization and recognition. Noise, corruption, variations in handwriting, non-standard page layouts and notations are common problems affecting especially the centuries-old manuscripts.

Starting from a facsimile, the current state-of-the-art methods binarize the image, detect and group the staff lines, then remove the staff lines and classify the remaining symbols imposing rules and prior knowledge to obtain the final digital representation. The first steps are critical for the performance of the overall system.

Here we propose to handle binarization, staff detection and noise removal by means of dynamic programming (DP) formulations. Our main insights are: a) the staves (the 5 -groups of staff lines) are represented by repetitive line patterns, are more constrained and informative, and thus we propose direct optimization over such patterns instead of first spotting single staff lines, b) the optimal binarization threshold also is the one giving the maximum evidence for the presence of staves, c) the noise, or background, is given by the regions where there is insufficient stave pattern evidence. We validate our techniques on the CVC-MUSCIMA(2011) staff removal benchmark, achieving the best error rates (1.7\%), as well as on various, other handwritten score facsimiles from the Renaissance.
\end{abstract}

\section{Introduction}

There is a growing interest in the automated digitization and transcription of handwritten music score facsimiles $[1,2]$. Of particular interest are old musical manuscripts, to be brought into a modern form as to prevent their music from being forgotten.

Most of the state-of-the-art Optical Music Recognition (OMR) systems share the same processing steps. First, operations such as enhancement, contrast stretching, or color normalization are applied. Second, a binarized image is obtained by means of local content-adaptive or global color thresholding. Third, the staff lines are detected and removed. Fourth, the musical symbols are segmented and classified. The final step typically combines symbol recognition with relative stave positioning, often exploiting music-specific rules. At that point, the transcription into modern formats (which can very well be digital, like MIDI) is possible. Obviously, the first processing steps are critical for the overall OMR performance. 
As a matter of fact, the current OMR systems have serious difficulties in dealing with handwritten scores and especially those with non-standard page layouts and deviating, century-old notations (e.g. Renaissance). Noise, corruption and variations in handwriting are other common issues that one OMR needs to address.

We contribute an automatic process for stave discovery based on the accumulated evidence of staves as 5-groups of staff lines, integrating binarization, stave and staff line detection, staff line and background removal.

We propose to handle binarization, staff line detection and noise removal by means of dynamic programming (DP) formulations. Our main insights are: a) the staves (the 5-group of staff lines) are more constrained and therefore more informative, and thus we propose a direct optimization over such patterns instead of first spotting single staff lines, b) the optimal binarization threshold also is the one giving the maximum evidence of the presence of staves, c) the noise or background, i.e. regions not containing musical score information, corresponds to the regions without sufficient evidence for the presence of stave patterns.

Binarization of musical score facsimiles aims at the accurate separation of the musical information (staves and musical symbols) from the paper support (background). The most employed techniques are Otsu thresholding and local adaptive thresholding over the grayscale image [3]. While there were many attempts at improving these basic thresholding schemes, few explicitly use the musical content for driving the binarization process. We will assume that the best separation is achieved when we have the strongest cumulated evidence for staves as repetitive line patterns. The approach of [4] probably comes closest and uses the distribution of vertical run-lengths of paired subsequent black and white segments to select the best gray-level threshold. Those paired run-lengths provide the most likely staff line thickness and following spacing. Our approach, on the other hand, does not stop at global statistics of run-length codes, but computes the local constrained stave evidence, thus more strongly exploiting the musical score properties. The best cumulated stave evidence for an image is spotting the best parameters for automatic stave discovery: gray-level threshold, staff line thickness, and inter-line spacing. A more detailed description comes later.

Staff detection and removal aims at localizing the staves as major components for musical score documents and at accurately removing the staff lines as to ease musical symbol recognition. The defining characteristics of staffs are their line thickness and the inter-line spacing within the staves. The detection of staff lines is affected by the fact that in most real musical facsimiles they exhibit different kinds of deformations such as bending, interruptions, pixel noise, intersections with other symbols, and deviations from constant line thickness and spacing. Horizontal and vertical projections $[5,6]$ are simple ways to detect staff lines when the deformations are (very) small. More robust approaches are based on line tracking $[7,8]$, staff segments [9,10], Skeleton fitting [1], stable path search [2] and staff line shape determination [11]. 
The removal techniques assume a prior detection step was performed to then remove the staff lines. They focus on the intersections with other musical symbols, as well as on the local line thickness. The algorithms can be grouped into those that apply line tracking, vector fields, run-lengths and skeletonization [1].

Most staff detection algorithms are based on the 3 steps of spotting the staff lines individually, staff line grouping into staves, and stave validation. Our approach, on the other hand, considers each stave as a whole, i.e. as an equi-distant repetition of staff lines. These patterns are important to impose context, through the power of the entire group of staff lines. The stave patterns are likely to show up in vertical slices through the score image. After detecting such local evidence, a dynamic programming formulation links up such evidence, crossing the page from left to right and also from right to left. The cumulation of the evidence should ideally lead to the same stave twice. Finally, the horizontal extent of the staves is determined, i.e. until where exactly they run, which includes finding possible interruptions breaking up the staves. To that end, a second dynamic programming algorithm makes the distinction between staves and background.

Note that the $\boldsymbol{u}$ se of $\boldsymbol{D P}$ is not novel for staff detection. The stable path [2] and the candidate point matching [10] techniques most resemble our DP formulation for staff detection. The stable paths [2] are in fact iteratively extracted shortest paths (and thus individual staff line candidates) over a built graph representation of the binarized image, after which they are grouped into staves. The candidate point matching technique [10] is optimizing the assignment of staff line candidate points in neighboring vertical scan lines using a penalized edit distance formulation, thus the potential staff lines are propagated across the vertical scan lines. We, on the other hand, impose a pattern (group of lines) for stave (and not staff line!) detection and optimize in a DP formulation the evidence for such stave propagation across the vertical scan lines. Thus, our DP formulation uses patterns instead of points and accumulates the highest possible evidence for complete staves.

In summary, our contributions are: a stave-based staff detection method and principled ways for automatic binarization and stave parameter discovery.

We validate our techniques on the CVC-MUSCIMA (2011) staff removal benchmark [12], achieving the best error rates (1.7\%) and on various handwritten score facsimiles from the Renaissance.

The structure of the remainder of the paper is as follows. Section 2 introduces our proposed methods for binarization and staff detection and removal focusing on the most innovative aspects. Section 3 describes the experimental setup and discusses the obtained results. Finally, the conclusions are drawn in Section 4.

\section{Proposed Method}

The overall procedure of our proposed binarization and staff line detection and removal technique is depicted in Fig. 1. First, for each threshold and the corresponding binarized image, stave evidence is summed for various staff line thicknesses and staff inter-spacings. The best evidence is considered to represent the 


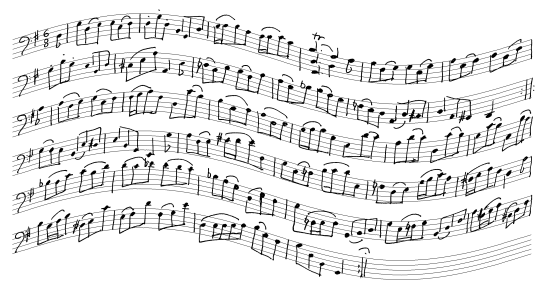

a) binarization

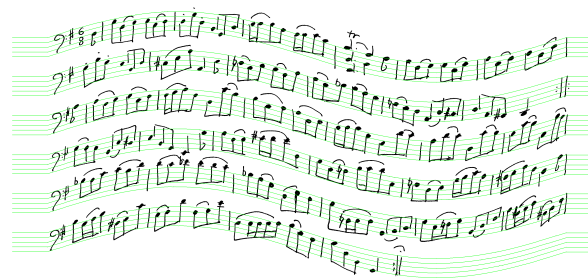

c) staff line detection

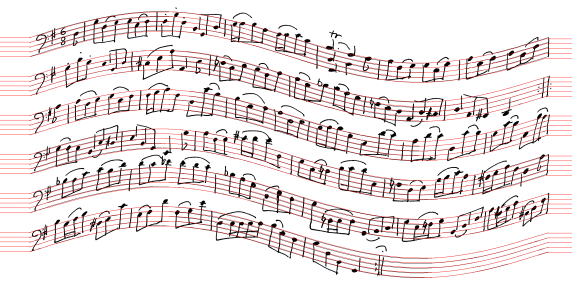

b) stave detection

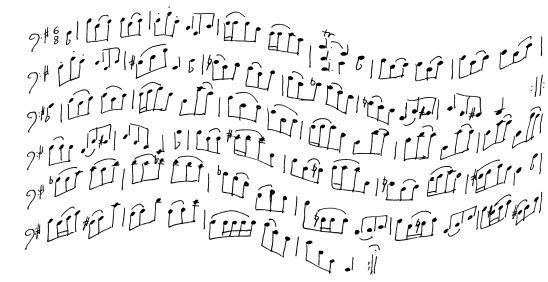

d) staff line removal

Fig. 1. Processing steps on a CVC-MUSCIMA deformed score.

real stave content of the image. Second, for the best global threshold and estimated staff line thickness and staff spacing we trace back the staves. Third, for each traced stave proposal we post-label it into stave information and background, thus segmenting the musical content from the surroundings. At last, the final image with musical symbols is obtained after the removal of the detected staff lines. The accurate staff line detection is necessary since the loose stave model does not fit precisely the staff lines. In Fig. 1 is shown that the red lines predicted by stave detection step are less precise than the green lines from the accurate staff detection step, in following the black staff lines.

In the rest of this section we focus on stave detection using the DP formulation, then on how the summed stave evidence is used for automatic discovery of stave parameters and the grayscale threshold for binarization, and finally we describe the staff line removal part.

\subsection{Stave Detection}

The input of our stave detection procedure is a binary musical document. The staff lines and musical symbols are marked with ' 1 ', while the background is ' 0 ' (see Fig. 2a). The staff line thickness $(\alpha)$ and staff line spacing $(\beta)$ are considered fixed for the moment. Section 2.4 describes how they are determined.

Along each of a number of regularly spaced vertical page sections, corresponding to the image's columns, a pixel-wise stave evidence score is calculated for the black-and-white pattern just below. This score quantifies the degree to which 5 black runlengths of more or less equal length are alternating with white runlengths, also of equal length. Such runlength sequence is illustrated in Fig. 2b. 


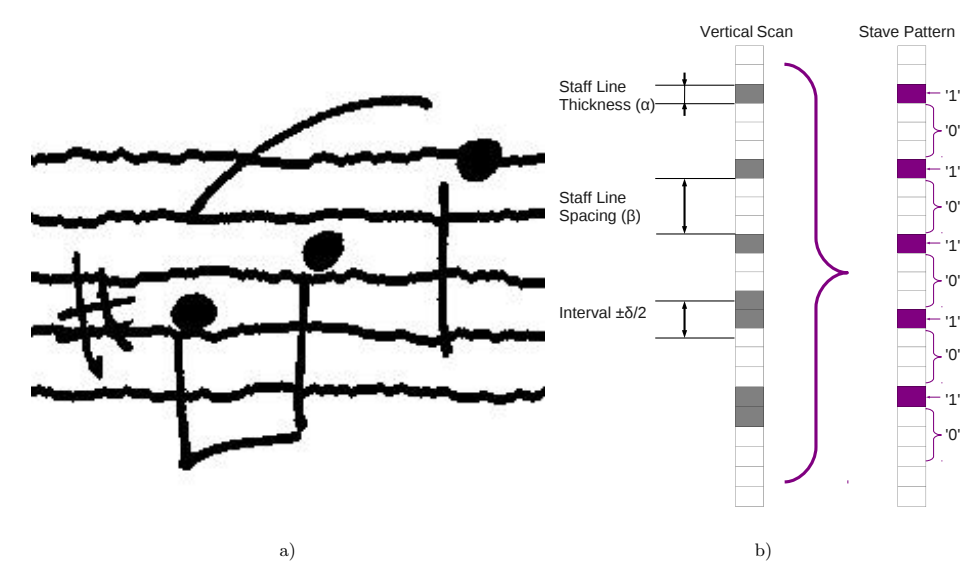

Fig. 2. Stave and pattern example.

The ideal situation is shown in the figure on the right, but, of course, in reality one has to allow for some slack on these lengths, as shown on the left and as discussed later when we describe the staff line removal process. In any case, we assume that each staff line is at least one black pixel (' 1 ') thick and, similarly, that each inter-staff spacing is at least one white pixel (' 0 ') thick. As to the allowable tolerance in the relative positions of the staff lines, we assume that there can be an independent jitter on each. The need for this tolerance is illustrated in Fig. 2a. Each of the staff lines are assumed to lie within an interval symmetrical about their nominal position (i.e. when following a strict succession of intervals with width $\alpha$ and $\beta$ ). The width of this jitter interval is given by $\delta$, defined as

$$
\delta=\min \{2 \times \alpha, \alpha+\beta-2\}
$$

We subtract 2 to avoid counting 2 neighboring black pixels as evidence for 2 neighboring staff lines. We validate this insight experimentally on several scores. A similar formula is used for the maximum staff line thickness, see equation (7).

For a point at image position $(i, j)$, the local stave evidence score is computed as follows. Let $M$ be the binarized image, then the local stave evidence $v(i, j)$ at position $(i, j)$ of our pattern of $K$ staff lines (always $K=5$ in our case) is given as:

$$
\begin{aligned}
v(i, j)= & \sum_{k=0}^{K-1} \max _{t \in\{-\lfloor\delta / 2\rfloor, \cdots,\lfloor\delta / 2\rfloor-1\}} M(t+i+k L, j)+ \\
& \sum_{k=0}^{K-1}\left(1-\min _{t \in\{\lfloor\alpha / 2\rfloor, \cdots, L-\lfloor\alpha / 2\rfloor-1\}} M(t+i+k L, j)\right)
\end{aligned}
$$

where $\alpha$ is the staff line thickness, $\beta$ is the staff spacing, $L=\alpha+\beta$. This score counts the number of times the nominal positions of staff lines and spacings coincide with the corresponding black and white regions, resp. Each such match is only counted once. When also taking a spacing below the lowest staff line, 
the maximum score is $2 K$. At first glance $v(i, j)$ is a very permissive pattern evidence score, but later, when using the context information under the form of the stave evidence in pixels from the neighboring columns, extra smoothing constraints will be imposed for propagating the global stave evidence.

In the next step, we integrate the local stave evidence scores across the vertical sections (columns). A dynamic path search algorithm accumulates local stave evidence scores, trying to keep that sum maximal, while moving from left to right. Note that the vertical scan lines impose a strict order, not allowing for the path to meander heavily. Moreover, in this left-to-right propagation a smoothing constraint is applied that penalizes vertical displacements. When selecting a pixel $i$ in the next column $j$, the recursive DP evidence is obtained as

$$
\begin{aligned}
& c(i, 1)=v(i, 1), \\
& c(i, j)=v(i, j)+\max _{k \in\{-2,-1,0,1,2\}}(c(i+k, j-1)-|k| \gamma)
\end{aligned}
$$

where $\gamma$ is the penalty set to $\lceil 2 K+K / 2\rceil=13$ and empirically validated on several scores.

Tracing back we obtain the candidate stave paths with the highest evidence. Applying a second pass of the stave detection DP algorithm in reverse order of the scans, allows us to detect the stable paths as in [2]. That is, those paths that regardless of the direction of the DP computation accumulate the same evidence. These stable paths are our stave detections. The procedure can be repeated after masking the already detected staves to extract staves with lower evidence. Note that the number of repetitions needed is bounded by the worst case number, given by the number of rows of $M$ divided by $K(\alpha+\beta)$, where $K$ is the number of staff lines in the stave pattern. In practice, for most of the binarized images we run up to 2 iterations to find all the staves. The technique assumes that one stave path always connect from the first to the last column. If the staves are vertical, or close to, this is detected and the score is rotated such that the staves are horizontal and the assumption holds for applying the technique. Since the technique handles well large rotations, for uncontrolled scores, the best is to run the whole stave discovery process for both original and 90 degrees rotated score and pick the rotation with the largest cumulated stave evidence.

\subsection{Stave segmentation}

Once we have detected a stave (see Section 2.1), under the form of a path connecting the first up to the last column, we want to segment the real musical content (stave and musical symbols) from the surrounding background. We remind the reader that a path (stave detection) connects single pixels at positions $p_{j}$ from each column $j$ in an ordered way.

We reuse the local stave evidence scores $(v(i, j))$ and the smoothness as implemented previously. Moreover, we impose prior knowledge under the form of a minimum stave segment width $\left(\lambda_{1}\right)$ and a minimum background segment 
width $\left(\lambda_{0}\right)$. In this way we avoid segments that are too short. Thus, we have a two-class labeling problem ('stave' $=$ ' 1 ' and 'background' $=$ ' 0 '). The recursive DP formulation for the stave segmentation is:

$$
\begin{aligned}
d\left({ }^{\prime} 1^{\prime}, 1\right)= & v\left(p_{1}, 1\right)-\theta_{1}, \\
d\left({ }^{\prime} 0^{\prime}, 1\right)= & \theta_{0}-v\left(p_{1}, 1\right), \\
d\left({ }^{\prime} 1^{\prime}, j\right)= & \max \left\{v\left(p_{j}, j\right)-\theta_{1}+d\left({ }^{\prime} 1^{\prime}, j-1\right)-\left|p_{j}-p_{j-1}\right| \gamma,\right. \\
& \left.\sum_{i=j}^{i=j-\lambda_{0}+1}\left(\theta_{1}-v\left(p_{i}, i\right)\right)+d\left({ }^{\prime} 0^{\prime}, j-\lambda_{0}\right)\right\} \\
d\left({ }^{\prime} 0^{\prime}, j\right)= & \max \left\{\theta_{0}-v\left(p_{j}, j\right)+d\left({ }^{\prime} 0^{\prime}, j-1\right),\right. \\
& \left.\sum_{i=j}^{i=j-\lambda_{1}+1}\left(v\left(p_{i}, i\right)-\theta_{0}\right)+d\left({ }^{\prime} 1^{\prime}, j-\lambda_{1}\right)\right\}
\end{aligned}
$$

where for each label class we empirically set the thresholds deciding where it is more likely to have staves and where not as $\theta_{1}=9.5$ and $\theta_{0}=7$. We are taking $\lambda_{0}=2 \times(\alpha+\beta)$ tolerating background segments no shorter than twice the staff height (thickness plus spacing), and $\lambda_{1}=5 \times(\alpha+\beta)$, thus tolerating stave segments no shorter than the height of the stave (a 5 -group of staff lines).

Tracing back for the best label solution gives us the optimal segmentation.

\subsection{Accurate Staff Detection}

In practice, the currently extracted staff line trajectories and the subsequent musical content (staves) segments are not precise enough (see Fig. 1b) to apply staff line removal. In this section, we refine such data. For this purpose, we use the current stave detections to define corridors where we can search for the accurate positions of the staff lines. The corridors are $\alpha+\beta$ wide and centered on the staff line position predicted by the stave detection. In this step, the different staff lines are refined more individually.

The local evidence $w$ of staff line existence at pixel $(i, j)$ inside the corridors is calculated as follows. A column template corresponding to a group of three staff lines with nominal width $\alpha$ and $\beta$ spacings in between is considered. This template is positioned with its center within the staff line corridor. Beneath the three staff line intervals (each of width $\alpha$ ) the system looks for black pixels. Each of these pixels contributes with their own weight, with emphasis on those in the middle staff interval. Moreover, within an interval of $\delta$ high around the middle staff interval, but excluding the latter, white pixels are sought. More precisely, the local evidence is now calculate as:

$$
\begin{aligned}
w(i, j) & =\sum_{k=i-\lfloor\alpha / 2\rfloor}^{i+\lfloor\alpha / 2\rfloor}(\sigma M(k, j)+M(k-(\alpha+\beta), j)+M(k+(\alpha+\beta), j)) \\
& +\sum_{k=1}^{\lfloor\delta / 2\rfloor}(2-M(i-\lfloor\alpha / 2\rfloor-k, j)-M(i+\lfloor\alpha / 2\rfloor+k, j))
\end{aligned}
$$


where $\delta$ is given by eq. (1) and we empirically set $\sigma=3$. This procedure is repeated with the template moved within the corridors of each single staff line. For the pixels $(i, j)$ outside the corridors or at their boundaries $w(i, j)=-\infty$.

The evidence propagation is solved using the same DP formulation from stave detection:

$$
\begin{aligned}
& f(i, 1)=w(i, 1), \\
& f(i, j)=w(i, j)+\max _{k \in\{-2,-1,0,1,2\}}\left(f(i+k, j-1)-|k| \gamma_{s}\right)
\end{aligned}
$$

with a different $\gamma_{s}$ as penalization term enforcing smoothness. In our experiments $\gamma_{s}$ is set to half from maximum local evidence, $\gamma_{s}=0.5(\delta+\alpha(\sigma+2))$.

Note that we can solve the evidence propagation for all the corridors in one pass through the image. Tracing back the best cumulated evidence path along each corridor provides the accurate staff line detection (see Fig. 1c).

\subsection{Stave Parameter Determination}

The main characteristics for a stave are the number of staff lines ( 5 lines are common for Western musical scores), the staff line thickness $(\alpha)$, and the interstaff spacing $(\beta)$. We propose to use the overall stave pattern evidence to drive the process of fitting the best thickness and spacing. For this, we start from an interval for $L$, defined as the sum of these two parameters and we assume that we have stave patterns on a page. Then, we start the search for the optimal values in a crude fashion. First we investigate the possible $(\alpha=\beta$ or $\alpha+1=\beta)$ and $L=\alpha+\beta$. The $L$ values for which the stave detection returns a sufficiently high overall evidence are then considered in more detail. For those values, we test all combinations $\alpha \in\{1, \cdots, L / 2\}$ and $\beta=L-\alpha$ and pick the combination returning the highest overall evidence.

\subsection{Threshold Selection for Binarization}

At the start of the algorithm, we look for a global threshold, applied to the intensity values (and not to color, similar to [3]). This thresholding operation is supposed to split music symbols and staves from background. In reality, parts of the illustrations and background blotches will end on the music side. Whereas Pinto et al. [3] consider vertical run lengths to drive the search, we once more use the staff evidence as computed using the stave detection procedure, applied with different threshold candidate values (see sections 2.1 and 2.4). It is assumed that the best evidence is achievable using the proper set of parameters (threshold, staff line thickness and staff spacing). For a chosen threshold, we extract staves, then compute the staff line evidence for all the detected staves. Summing up for all staff lines we have a staff evidence at score level. We offer the insight, verified by experiments, that the best such evidence is achieved for the proper binarization threshold. As with $\alpha$ and $\beta$ not all threshold values need to be tested. An Otsu threshold extracted from the entire image tends to narrow down the range of potential threshold values quite effectively. 

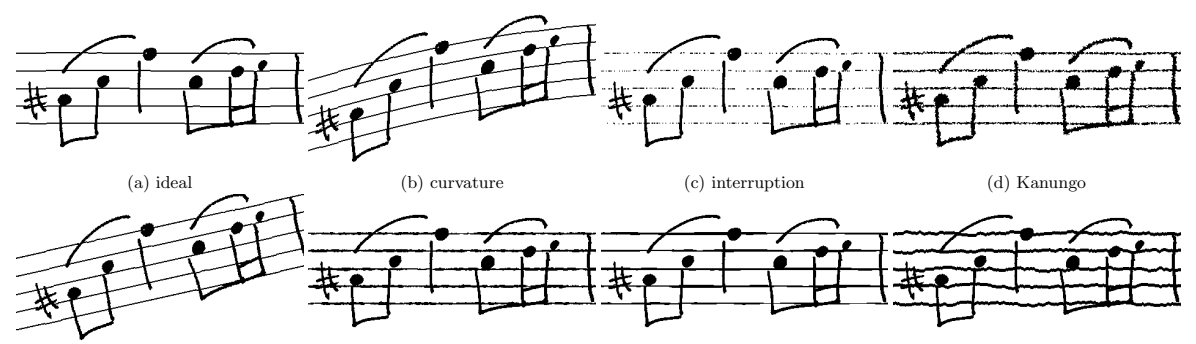

(e) rotatio

(f) line-thickness-v1

(g) line-thickness-v2

(h) line-y-v1

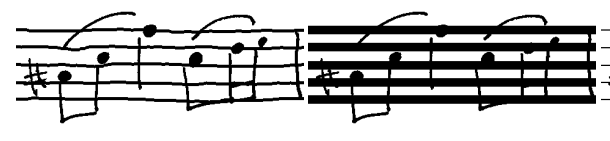

(i) line-y-v2

(j) thickness-ratio

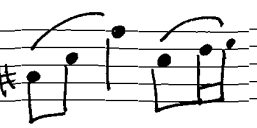

(k) typeset-emulation

(1) whitespeckles

Fig. 3. Deformation types in CVC-MUSCIMA benchmark.

\subsection{Staff Line Removal}

After stave discovery and accurate staff line detection, we remove the staff line pixels to serve a possibly following music symbol classification with a cleaner set of symbols. The problems come from intersections between the staff lines and those symbols, and from the fact that the detected staff lines do not match the real lines exactly, due to small errors or interpolation issues. The interpolation issues are caused by the fact that DP picks the shortest path for interpolation across gaps in the stave/staff evidence, which may not be the one with the curvature of the flanking portions. However, if not all staff lines have a gap simultaneously, the complete ones will drive the course of the interpolated ones.

Here we use one of the simplest ways to handle the staff line removal. It follows the idea from [11]. The vertical segments intersecting the detected staff line are considered to belong to the line if the segment length is less than the maximum staff line thickness. Otherwise, they are considered part of the music symbols. The maximum staff line thickness is empirically set to:

$$
\delta=\min \{2 \times \alpha, \alpha+\beta\}
$$

\section{Experiments}

\subsection{Parameter Setting}

The presented automatic stave discovery method encompasses several distinct problems with specific algorithmic solutions. One can note that we have a large number of parameters. The parameters can be categorized into fixed $(K)$, automatically determined $(\alpha, \beta, L)$, derived from those $\left(\delta, \gamma, \lambda_{0}, \lambda_{1}\right)$, and manually tuned $\left(\gamma_{s}, \theta_{0}, \theta_{1}, \sigma\right)$.

Thus, the number of staff lines per stave is set to $K=5$ for the musical scores considered here. The binarization threshold, staff line thickness $\alpha$, spacing $\beta$ and $L=\alpha+\beta$ are automatically determined. The maximum bound $(\delta)$, the penalty 
$(\gamma)$, the minimum allowed stave segment width $\left(\lambda_{1}\right)$ and background segment width $\left(\lambda_{0}\right)$, depend solely on the fixed and automatically determined parameters. However, the user only needs to set the penalty $\gamma_{s}$, the likeliness thresholds $\left(\theta_{0}\right.$, $\theta_{1}$ ) and the weight $\sigma$ which depend on the noise and conditions from the score images used as input. While we have tried automatic methods for adaptively set these parameters to different input conditions, we do not have yet a general solution.

\subsection{Staff Detection and Removal}

The proposed method is qualitatively evaluated on real Renaissance facsimiles ${ }^{1}$ and quantitatively evaluated for staff removal on the CVC-MUSCIMA benchmark [12] as used during the competition held at ICDAR 2011 [13]. The dataset consists of 1,000 handwritten music score images from 50 writers and the images are (synthetically) distorted using 11 deformation models (see Fig. 3). Each deformation model generates 1,000 images. In total, the dataset contains 12,000 images equally divided into training data and testing data. These images are already binarized. The raw images are not available. For each of the binarized images the dataset also provides the ground truth binary image with the staff lines removed. To the best of our knowledge, it is the largest benchmark for staff line removal evaluation.

Our results are evaluated using the pixel based metric as used for the competition, where the error rate is computed as described in $[1,13]$ :

$$
\text { E.R. }=100 \times \frac{\# \text { misclassified } s p+\# \text { misclassified non } s p}{\text { \#all } s p+\# \text { all non } s p}
$$

where \# means "number of" and $s p=$ stands for "staff line pixels". Only the musical content pixels are considered for classification ('black'/'1' pixels in our paper, see Fig. 1a).

First, in Table 1, we show quantitative results of our method on the competition training dataset. Thus, we compare with the results reported by [11] for this training (!) set. Our method performs very well for each kind of deformation, which is not the case for the reference method [11], which performs weakly on the 'large thickness ratio' images. The average error rate is $1.5 \%$, which compares favorable even when the other method does not account for the failure cases.

Second, in Table 1 we provide the more relevant results for the competition testing dataset and compare also with the top 3 performing methods (out of 8 entries) [13,11]. ISI01-HA is the winning method of the competition [13], NUG04-LTr is the line tracking method [1] and INP02-SP is the stable path method of [2]. Our proposed method performs the best overall, works for all kinds of deformations (like ISI01-HA does), and clearly improves over the stable path method (INP02-SP). The stable path method follows a DP formulation based on iteratively computing shortest paths (thus potential staff lines) and post grouping these lines into staves. We, on the other hand, directly compute

\footnotetext{
${ }^{1}$ Qualitative results are included in the supplementary material.
} 
Table 1. Results comparison on CVC-MUSCIMA staff removal dataset

\begin{tabular}{|c|c|c|c|c|c|c|c|}
\hline \multirow[b]{3}{*}{ Deformation Type } & \multicolumn{7}{|c|}{ Error Rates (\%) } \\
\hline & \multicolumn{5}{|c|}{ Testing data } & \multicolumn{2}{|c|}{ Training data } \\
\hline & [11] & NUG04-LTr & ISI01-HA & INP02-SP & Ours & [11] & Ours \\
\hline Ideal & & 2.08 & 1.50 & 1.50 & 1.34 & 1.33 & \\
\hline Curvature & & 100 & 1.66 & 1.80 & 1.34 & 1.43 & \\
\hline Interrupted & & 100 & 0.91 & 6.1 & 0.84 & 1.02 & \\
\hline Kanungo & & 4.33 & 2.84 & 2.86 & 2.59 & 2.84 & \\
\hline Rotation & & 100 & 1.76 & 2.03 & 1.65 & 1.65 & \\
\hline Line Thickness-variation-v1 & & 3.74 & 2.17 & 2.70 & 1.85 & 3.62 & \\
\hline Line Thickness-variation-v2 & & 3.74 & 2.15 & 3.01 & 1.90 & 2.89 & \\
\hline Staff Line-y-variation-v1 & & 5.94 & 1.89 & 2.43 & 1.51 & 4.58 & \\
\hline Staff Line-y-variation-v2 & & 3.73 & 1.83 & 2.27 & 1.40 & 3.64 & \\
\hline Thickness ratio & $\mathrm{N} / \mathrm{A}$ & 10.78 & 2.86 & 6.89 & 4.05 & $\mathrm{~N} / \mathrm{A}$ & \\
\hline Typeset-emulation & & 4.83 & 1.60 & 1.60 & 1.34 & 2.09 & \\
\hline White speckles & & 1.76 & 1.48 & 1.73 & 1.39 & 1.37 & \\
\hline Overall Error Rate & 1.95 & 28.41 & 1.89 & 2.91 & 1.76 & 2.41 & 1.57 \\
\hline
\end{tabular}

the staves as entities, using a DP formulation, rather than individual staff lines. The results show that this is beneficial. If we do not employ the accurate staff detection step in our pipeline the performance significantly degrades and the overall error rate increases from $1.7 \%$ to $3.1 \%$ on the testing set.

\subsection{Binarization}

We qualitatively assess our proposed stave evidence driven binarization method on facsimiles from the Renaissance. In Fig. 4 we depict a difficult case with very low resolution (230x320), blur, and an increased amount of non-musical score content (pictures). Our method picks a grayscale global threshold level such that the stave evidence is maximized. On the other hand, Otsu's method finds a good separation of the image pixels into two classes, thereby not guaranteeing that this global threshold is reasonable to separate music symbols and staves from the non-uniform background. However, while our method is able to provide an optimal global threshold for stave evidence, it is not clear if the same threshold is optimal for musical symbol classification after staff line removal. The best solution for automated music transcription may therefore come out to first segment the musical content using the staves (as in Fig. 5d) and then to get the optimal threshold using also the musical symbols, not only the staff lines, for those parts surviving the first threshold. The global thresholding methods (such ours and Otsu's) were proved to perform worse than the local adaptive thresholding methods [3]. However, we did not quantitatively evaluate our global method against local methods.

\subsection{Renaissance Facsimiles}

The proposed automatic stave discovery method is qualitatively evaluated on hundreds of handwritten facsimiles from the Renaissance. ${ }^{2}$

\footnotetext{
${ }^{2}$ Qualitative results are included in the supplementary material.
} 


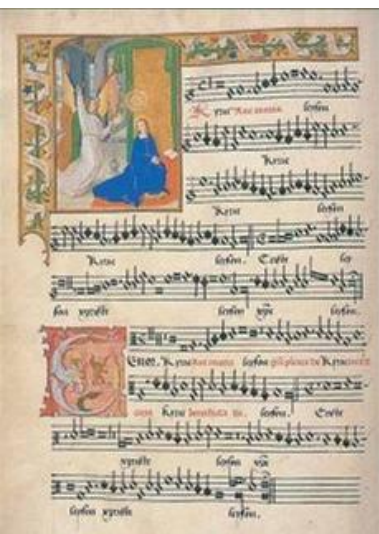

a) facsimile $(230 \times 320$ pixels $)$

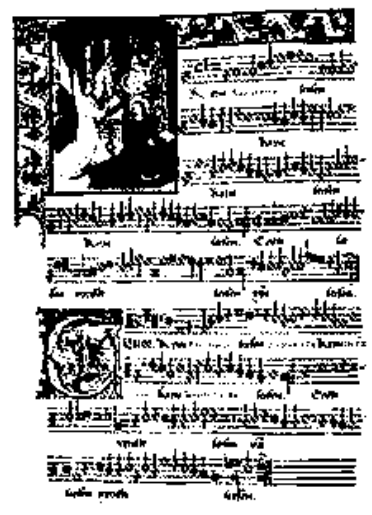

b) Otsu method

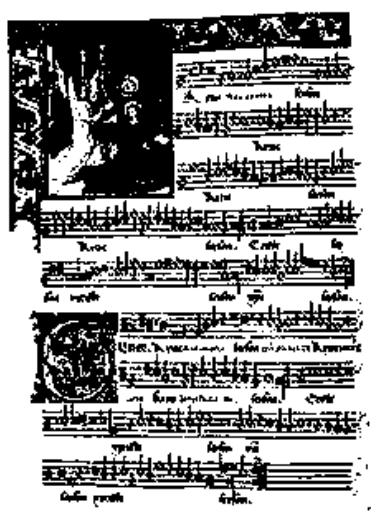

c) Ours

Fig. 4. Binarization example.

Fig. 5 shows partial results during the automated stave discovery process. This particular facsimile has low resolution $(858 \times 600)$, quite some distortions due to the non-planar paper support, residual noise transpiring from the other side of the paper, the omnipresent pictures and text, and the variations in the handwriting. Moreover, the musical content region is relatively small w.r.t. the whole image space and there are two columns of staves with different lengths.

The stave discovery led to a visually meaningful binarization (Fig. 5b) with an excellent (and correct) stave detection as well (see Fig. 5c), the blue-green lines). The segmentation into musical content (green colored segments in Fig. 5c) and non-musical content (blue colored segments in Fig. 5c) is able to cope with large gaps between the staves and with the presence of noise. Moreover, the final content segmentation keeps most of the musical information (see Fig. 5d).

\subsection{Limitations and Further Research}

The proposed staff line detection and removal method is robust and effective for different kinds of deformed musical facsimiles, but it has some limitations.

First, the method does not interpolate the missing evidence (interruptions) in the facsimile, but rather tends to find the shortest path in an unnatural way. Using the path line extrapolation as extra smoothness term, shape line priors, or post-smoothing by line interpolation could mitigate this problem.

Second, the staff line removal criteria use only the staff line thickness, but context and crossings information should improve the performance.

Third, while we made a large step forward into automated binarization, parameter selection, and staff line/noise removal, the running time is linearly dependent on the number of pixels, which can be very large. A multi-scale strategy is desirable, starting with the DP optimization at coarser levels and only adding details to the finer levels. The running time of our single-thread CPU C ++ unoptimized code for stave detection, accurate staff detection and staff line removal is, on a Core 2 Quad 2009 desktop, on average less than 1 second per binarized 

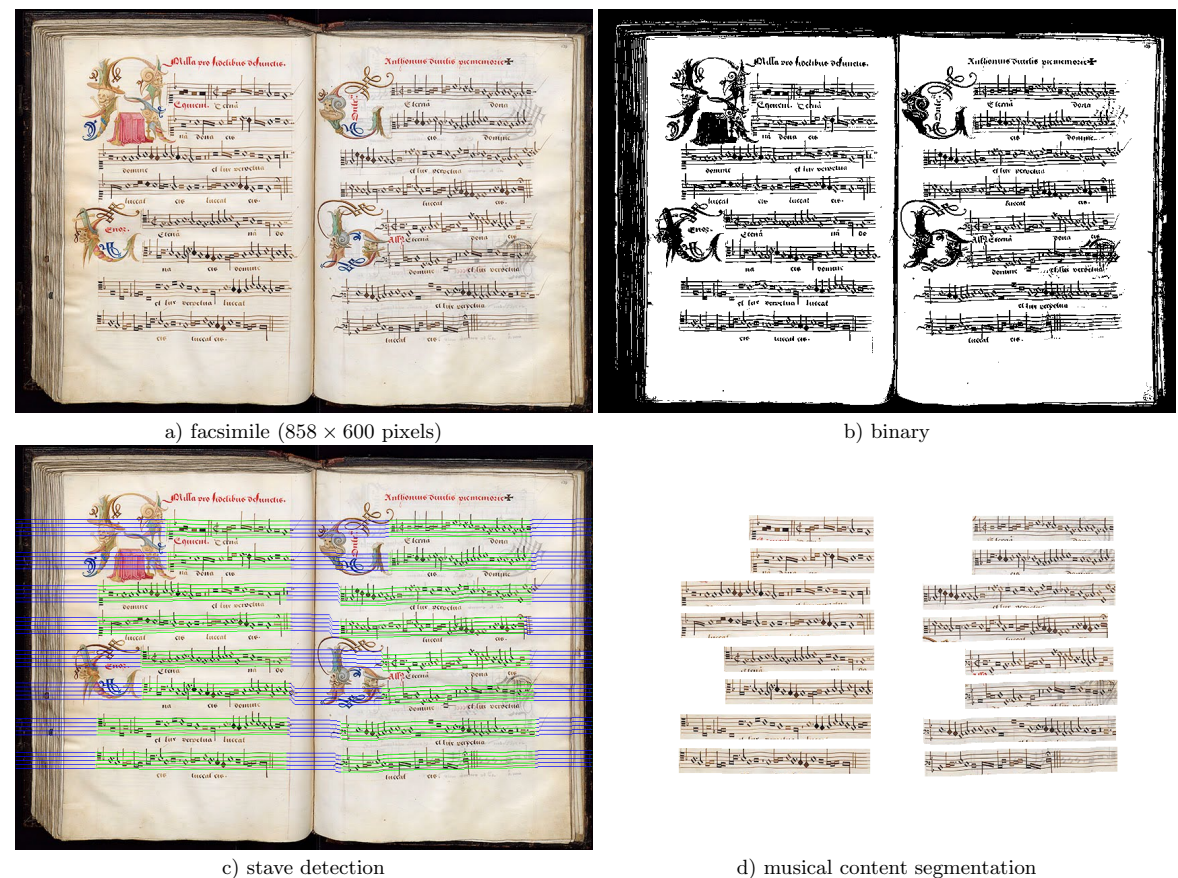

d) musical content segmentation

Fig. 5. Renaissance facsimile example.

image in the CVC-MUSCIMA dataset, where the average image is $\sim 2000 \times 3500$ pixels.

While relatively few work on this automatic stave discovery problems, this goes for many other applications. But exposing the community to applications is inspiring. Moreover, the problems dealt with are akin to wider issues, like text page layout analysis. One can think of an extension to handwritten documents with similar ruled but irregular structure.

\section{Conclusions}

A novel DP-based music staff line detection and removal technique for musical documents has been proposed. It uses the fact that the staff lines are grouped into staves following constrained patterns. Vertical slices of the document can reveal the stave patterns. Using DP we can propagate in one pass the stave evidence from one side of the document to another. The best staves are generated by tracing back the solutions. Another DP formulation optimally solves the labeling of such tracked solution into background and stave segments. This leads to accurate stave / background segmentations and thus noise removal. Also, we propose the maximum evidence criterion for binarizing the images. That is, we made the successful assumption that the staves will accumulate the largest evidence for the best binarized musical score image. The parameters of a stave such as staff line thickness and spacing were derived in the same way. 
The proposed technique is the top performer on the latest musical staff line removal benchmark under various deformations. Also, the techniques exhibit robustness and effectiveness on low quality facsimiles with Renaissance handwritten musical scores.

Acknowledgement. This work was supported by the Flemish IWT/SBO project ALAMIRE.

\section{References}

1. Dalitz, C., Droettboom, M., Pranzas, B., Fujinaga, I.: A comparative study of staff removal algorithms. IEEE Trans. Pattern Anal. Mach. Intell. 30 (2008) 753-766

2. dos Santos Cardoso, J., Capela, A., Rebelo, A., Guedes, C., da Costa, J.P.: Staff detection with stable paths. IEEE Trans. Pattern Anal. Mach. Intell. 31 (2009) 1134-1139

3. Pinto, T., Rebelo, A., Giraldi, G.A., Cardoso, J.S.: Music score binarization based on domain knowledge. In: Pattern Recognition and Image Analysis - 5th Iberian Conference (IbPRIA). (2011) 700-708

4. Cardoso, J.S., Rebelo, A.: Robust staffline thickness and distance estimation in binary and gray-level music scores. In: 20th International Conference on Pattern Recognition. (2010) 1856-1859

5. Blostein, D., Baird, H.S.: A critical survey of music image analysis. In: Structured Document Image Analysis. (1992)

6. Fujinaga, I.: Staff detection and removal. In George, S.E., ed.: Visual Perception of Music Notation: On-Line and Off Line Recognition, Hershey: IGI Global (2004) $1-39$

7. Bainbridge, D., Bell, T.C.: Dealing with superimposed objects in optical music recognition. In: 6th International Conference on Image Processing and its Applications. (1997) 756-760

8. Randriamahefa, R., Cocquerez, J., Fluhr, C., Pepin, F., Philipp, S.: Printed music recognition. In: International Conference on Document Analysis and Recognition. (1993) 898-901

9. Dutta, A., Pal, U., Fornés, A., Lladós, J.: An efficient staff removal approach from printed musical documents. In: 20th International Conference on Pattern Recognition. (2010) 1965-1968

10. Miyao, H.: Stave extraction for printed music scores. In Yin, H., Allinson, N., Freeman, R., Keane, J., Hubbard, S., eds.: Intelligent Data Engineering and Automated Learning IDEAL 2002. Volume 2412 of Lecture Notes in Computer Science. Springer Berlin / Heidelberg (2002) 621-634

11. Su, B., Lu, S., Pal, U., Tan, C.L.: An effective staff detection and removal technique for musical documents. In: 10th IAPR International Workshop on Document Analysis Systems. (2012)

12. Fornes, A., Dutta, A., Gordo, A., Llados, J.: CVC-MUSCIMA: A ground-truth of handwritten music score images for writer identifcation and staff removal. International Journal on Document Analysis and Recognition (2011) 1-9

13. Fornés, A., Dutta, A., Gordo, A., Lladós, J.: The ICDAR 2011 music scores competition: Staff removal and writer identification. In: ICDAR. (2011) 1511-1515 